\title{
Projection and contraction methods for constrained convex minimization problem and the zero points of maximal monotone operator
}

\author{
Yujing Wua , Luoyi Shib,* \\ a Tianjin Vocational Institute, Tianjin, 300410, P. R. China. \\ ${ }^{b}$ Department of Mathematics, Tianjin Polytechnic University, Tianjin 300387, P. R. China. \\ Communicated by Y. H. Yao
}

\begin{abstract}
In this paper, we introduce a new iterative scheme for the constrained convex minimization problem and the set of zero points of the maximal monotone operator problem, based on the projection and contraction methods. The core idea is to build the corresponding iterative algorithms by constructing reasonable error metric function and profitable direction to assure that the distance form the iteration points generated by the algorithms to a point of the solution set is strictly monotone decreasing. Under suitable conditions, new convergence theorems are obtained, which are useful in nonlinear analysis and optimization. The main advantages of the method presented are its simplicity, robustness, and ability to handle large problems with any start point. As an application, we apply our algorithm to solve the equilibrium problem, the constrained convex minimization problem and the split feasibility problem, the split equality problem in Hilbert spaces. (C)2017 All rights reserved.
\end{abstract}

Keywords: Fixed point, constrained convex minimization, maximal monotone operator, resolvent, variational inequality, split equality problem.

2010 MSC: 47H10, 54H25.

\section{Introduction}

Let $\mathrm{H}$ be real Hilbert space with the inner product $\langle$,$\rangle and norm \|\cdot\|$. Throughout the rest of this paper, I denotes the identity operator on Hilbert space $H$. If $T$ is an operator on $H, \operatorname{Fix}(T)$ denotes the set of the fixed points of an operator $T$ on $H$ (i.e., $\operatorname{Fix}(T)=\{x \in H, T x=x\}$ ). Assume $f: H \rightarrow R$ is a real-valued continuously differentiable function, let $\nabla f$ denote the gradient of the function $f$.

Firstly, assume $\Omega$ is a nonempty closed convex subset of $H$, consider the constrained convex minimization problem:

$$
\min _{x \in \Omega} f(x),
$$

where $f: \Omega \longrightarrow R$ is a real-valued convex function. However, we all know that the minimization problem (1.1) has more than one solution under some conditions, so regularization is needed in finding the unique

\footnotetext{
*Corresponding author

Email addresses: xiaomi20062008@163.com (Yujing Wu), shiluoyi@tjpu.edu.cn (Luoyi Shi)
} 
solution of the minimum normal solution of the minimization problem (1.1). Now, we consider the following regularized minimization problem:

$$
\min _{x \in \Omega} f_{1}(x)=\frac{1}{2}\|x\|^{2}+f(x)
$$

Assume consistency of the constrained convex minimization problem (1.2), and let $\Gamma_{1}$ denote the solution set of (1.2). It is well-known that if $f$ is differentiable, then the gradient-projection method generates a sequence $\left\{x_{n}\right\}$ by using the following recursive formula:

$$
x_{n+1}=P_{\Omega}\left[x_{n}-\lambda \nabla f\left(x_{n}\right)\right], \text { for } n \geqslant 1,
$$

or in a more general form:

$$
x_{n+1}=P_{\Omega}\left[x_{n}-\lambda_{n} \nabla f\left(x_{n}\right)\right], \text { for } n \geqslant 1,
$$

where $x_{1} \in \Omega$ is an arbitrary initial point and $\lambda, \lambda_{n}$ are positive real numbers.

In 2011, Xu [29] proved that if $\nabla \mathrm{f}$ is Lipschitz continuous and strongly monotone, then the sequence $\left\{x_{n}\right\}$ generated by (1.3), (1.4) can converge strongly to a minimizer of (1.2). If $\nabla f$ is Lipschitzian, then the schemes (1.2) and (1.3) can still converge weakly under certain assumptions. The algorithms for finding the approximate solutions of the constrained convex minimization problem has been studied by several authors, see for example $[3,4,7,14,23,28]$ and the references therein. The convergence of the sequence generated by this method depends largely on the behavior of the gradient of the objective function.

Secondly, consider the problem of zero points of maximal monotone operator, assume $A$ is a setvalued mapping from $\mathrm{H}$ to $2^{\mathrm{H}}$. $A$ is said to be a monotone operator on $\mathrm{H}$, if $\langle x-y, u-v\rangle \geqslant 0$ for all $x, y \in H, u \in A(x), v \in A(y)$. A monotone operator $A$ on $H$ is said to be maximal, if its graph is not properly contained in the graph of any other monotone operator on $\mathrm{H}$. The problem of zero points of maximal monotone operator is

$$
\text { finding } x \in H \text {, such that } 0 \in A(x) \text {, }
$$

where $A$ is a maximal monotone operator on $\mathrm{H}$. Some methods have been proposed to solve the problem of zero points of maximal monotone operator, for instance, [8, 9, 15, 17, 21, 24, 26].

In this paper, motivated and inspired by the above results, we propose the following constrained convex minimization and the set of zero points of the maximal monotone operator problem: Assume $f: \Omega \rightarrow R$ is a real-valued convex function and $I+\nabla f$ is $v$-inverse strongly monotone, $A: H \longrightarrow 2^{H}$ is a maximal monotone operator. Find $u^{*} \in \Omega$ such that

$$
\frac{1}{2}\left\|u^{*}\right\|^{2}+f\left(u^{*}\right)=\min _{u \in \Omega} \frac{1}{2}\|u\|^{2}+f(u), \quad \text { and } \quad u^{*} \in A^{-1}(0) .
$$

We use $\Gamma$ to denote the solution set of the problem (1.5) and assume consistency of this problem.

The paper is organized as follows: In Section 2 we collect some elementary concepts and facts which will be used in the proofs of our main results. In Section 3 we introduce a new projection and contraction iterative algorithm for the constrained convex minimization and the set of zero points of the maximal monotone operator problem and obtain the convergence theorem. Finally, in Section 4 we apply the above algorithm to the equilibrium problem, the constrained convex minimization problem and the split feasibility problem, the split equality problem in Hilbert spaces.

\section{Preliminaries}

We now collect some elementary concepts, facts and three impotent inequalities which will play an important role in projection and contraction methods.

Projections are an important tool for our work in this paper. Let $\mathrm{H}$ be real Hilbert space, the projection $\mathrm{P}_{\Omega}$ from $\mathrm{H}$ onto a nonempty closed convex subset $\Omega$ of $\mathrm{H}$ is defined by

$$
\mathrm{P}_{\Omega}(w)=\arg \min _{\mathrm{x} \in \Omega}\|x-w\| .
$$


It is well-known that $P_{\Omega}(w)$ is characterized by the inequality

$$
\left\langle w-P_{\Omega}(w), x-P_{\Omega}(w\rangle \leqslant 0, \quad \forall x \in \Omega .\right.
$$

Firstly, we introduce some other sorts of nonlinear operator.

Definition 2.1. Assume $\mathrm{T}: \mathrm{H} \longrightarrow \mathrm{H}$ is a nonlinear operator.

(a) $T$ is nonexpansive, if $\|T x-T y\| \leqslant\|x-y\|$ for all $x, y \in H$.

(b) $\mathrm{T}$ is averaged (av), if $\mathrm{T}=(1-\alpha) \mathrm{I}+\alpha \mathrm{S}$, where $\alpha \in(0,1)$ and $\mathrm{S}: \mathrm{H} \longrightarrow \mathrm{H}$ is nonexpansive.

(c) $T$ is $v$-inverse strongly monotone $\left(v\right.$-ism), with $v>0$, if $v\|T x-T y\|^{2} \leqslant\langle T x-T y, x-y\rangle$, for all $x, y \in H$.

(d) $\mathrm{T}$ is firmly nonexpansive (fne), if $\|T x-T y\|^{2} \leqslant\langle T x-T y, x-y\rangle$, for all $x, y \in H$.

The following lemma depicting the relationship between nonexpansive, av and $v$-ism.

Lemma 2.2 ([2]). If $\mathrm{T}: \mathrm{H} \longrightarrow \mathrm{H}$ is an operator, we have:

(a) $\mathrm{T}$ is nonexpansive, if and only if the complement $\mathrm{I}-\mathrm{T}$ is $\frac{1}{2}$-ism.

(b) $\mathrm{T}$ is av, if and only if the complement $\mathrm{I}-\mathrm{T}$ is $\mathrm{v}$-ism for some $v>\frac{1}{2}$.

(c) If $\mathrm{T}$ is $\nu$-ism and $\beta>0$, then $\beta \mathrm{T}$ is $\frac{\nu}{\beta}$-ism.

(d) Let $T_{1}$ and $T_{2}$ be av operators and suppose that $\operatorname{Fix}\left(T_{1}\right) \cap \operatorname{Fix}\left(T_{2}\right)$ is nonempty. Then $\operatorname{Fix}\left(T_{1}\right) \cap \operatorname{Fix}\left(T_{2}\right)=$ $\operatorname{Fix}\left(T_{1} T_{2}\right)=\operatorname{Fix}\left(T_{2} T_{1}\right)$.

It is well-known that the projection $P_{\Omega}$ is av and from [24], if $A$ is a maximal monotone operator and $\lambda>0, J_{\lambda}=(I+\lambda A)^{-1}$ the resolvent of $A$ for $\lambda>0$ is a single-valued operator and is fne. Furthermore, $A^{-1}(0)=\operatorname{Fix}\left(J_{\lambda}\right)$.

Secondly, we introduce the following two lemmas, which are well-known demi-closed principle and Opil's lemma, respectively.

Lemma $2.3([12,13])$. Let $\mathrm{X}$ be a Banach space, $\mathrm{C}$ be a closed convex subset of $\mathrm{X}$ and $\mathrm{T}: \mathrm{C} \rightarrow \mathrm{C}$ be a nonexpansive mapping with $\operatorname{Fix}(\mathrm{T}) \neq \emptyset$. If $\left\{\mathrm{x}_{\mathrm{n}}\right\}$ is a sequence in $\mathrm{C}$ weakly converging to $\mathrm{x}$ and if $\left\{(\mathrm{I}-\mathrm{T}) \mathrm{x}_{\mathrm{n}}\right\}$ converges strongly to $y$, then $(\mathrm{I}-\mathrm{T}) \mathrm{x}=\mathrm{y}$.

Lemma 2.4 ([20]). Let $\mathrm{H}$ be a Hilbert space and $\left\{w_{n}\right\}$ a sequence in $\mathrm{H}$ such that there exists a nonempty set $\Omega \subseteq \mathrm{H}$ satisfying the following items.

(i) For every $w \in \Omega, \lim _{n \rightarrow \infty}\left\|w_{n}-w\right\|$ exists;

(ii) any weak-cluster point of the sequence $\left\{w_{n}\right\}$ belongs to $\Omega$.

Then, there exists $\tilde{w} \in \Omega$ such that $\left\{w_{n}\right\}$ weakly converges to $\tilde{w}$.

Besides, the following lemma shows the close relationship between the constrained convex minimization problem and the variational inequality problem $\operatorname{VI}(\Omega, \nabla f)$.

Lemma 2.5 ([10]). Let $\mathrm{f}$ be a convex and differentiable functional and let $\Omega$ be a closed convex subset of $\mathrm{H}$. Then $x \in \Omega$ is a solution of the constrained convex minimization problem (1.2), if and only if $x \in \Omega$ satisfies the following variational inequality problem:

$$
\langle(I+\nabla f)(x), v-x\rangle \geqslant 0, \quad \forall v \in \Omega .
$$


At the end of this section, we will propose three impotent inequalities which will play an important role in projection and contraction methods.

The following proposition shows that the problem (1.5) is equivalent to a projection equation.

Proposition 2.6. Assume $\operatorname{Fix}\left(\mathrm{P}_{\Omega}\right) \cap \operatorname{Fix}\left[(1-\beta) \mathrm{I}+\beta\left(-\nabla f \mathrm{~J}_{\lambda}\right)\right] \neq \emptyset$ and $\operatorname{Fix}(-\nabla f) \cap \operatorname{Fix}\left(\mathrm{J}_{\lambda}\right) \neq \emptyset$, then $\mathrm{u} \in \mathrm{H}$ is a solution of the problem (1.5), if and only if $\mathrm{u}=\mathrm{P}_{\Omega}\left[\mathrm{u}-\beta\left(\mathrm{u}+\nabla f \mathrm{~J}_{\lambda}(\mathrm{u})\right)\right]$, for all $\beta \in\left(0, \frac{1}{2}\right)$.

Proof. Assume $u=P_{\Omega}\left[u-\beta\left(u+\nabla f J_{\lambda}(u)\right)\right]$, it is easy to see $u=P_{\Omega}\left[(1-\beta) u+\beta\left(-\nabla f J_{\lambda}(u)\right)\right]$. Since $P_{\Omega}$ is av and $\nabla f$ and $J_{\lambda}$ are all nonexpansive, one can obtain that $(1-\beta) I+\beta\left(-\nabla f J_{\lambda}\right)$ is av. By using Lemma 2.2 , we can get that

$$
u \in \operatorname{Fix}\left(P_{\Omega}\right) \cap \operatorname{Fix}\left[(1-\beta) I+\beta\left(-\nabla f J_{\lambda}\right)\right] .
$$

That is to say $u \in \Omega$ and

$$
(1-\beta) u+\beta\left(-\nabla f J_{\lambda}\right)(u)=u .
$$

So, $\left(-\nabla f J_{\lambda}\right)(u)=u$, i.e., $u \in \operatorname{Fix}\left(-\nabla f J_{\lambda}\right)$. Since $-\nabla f$ and $J_{\lambda}$ is av, by using Lemma 2.2 again, we have $u \in \operatorname{Fix}\left(J_{\lambda}\right)$.

Hence, $u=P_{\Omega}[u-\beta(u+\nabla f(u))]$. According to the property of projection $P_{\Omega}$, one can get

$$
\langle u-[u-\beta(u+\nabla f(u))], v-u\rangle \geqslant 0, \quad \forall v \in \Omega .
$$

That is,

$$
\langle\beta[\mathrm{I}+\nabla \mathrm{f}](\mathrm{u}), v-\mathrm{u}\rangle \geqslant 0, \quad \forall v \in \Omega .
$$

Using $u \in \operatorname{Fix}\left(\mathrm{J}_{\lambda}\right)$ and Lemma 2.5, one can obtain $u$ is a solution of the problem (1.5).

Conversely, assume that $u$ is a solution of the problem (1.5). By assumption, $u=J_{\lambda}(u)$ and $\langle[I+$ $\nabla \mathrm{f}](\mathrm{u}), v-u\rangle \geqslant 0$, for all $v \in \Omega$. Hence,

$$
\left\langle u-\left[u-\beta\left(I+\nabla f J_{\lambda}\right)(u)\right], v-u\right\rangle \geqslant 0, \quad \forall v \in \Omega, \quad \beta>0 .
$$

According to the property of projection $\mathrm{P}_{\Omega}$, one can get that

$$
u=P_{\Omega}\left[u-\beta\left(I+\nabla f J_{\lambda}\right)(u)\right], \quad \forall \beta>0 .
$$

Remark 2.7. Let $e(u):=u-P_{\Omega}\left[u-\beta\left(u+\nabla f J_{\lambda}(u)\right)\right]$, it follows that to solve the problem (1.5) is equivalent to find a zero point of the residue function $e(u)$.

Let $u^{*} \in \Gamma$ be a solution, we can get that $u^{*} \in \operatorname{Fix}_{\lambda}$ and $u^{*}=P_{\Omega}\left[u^{*}-\beta\left(u^{*}+\nabla f J_{\lambda}\left(u^{*}\right)\right)\right]$ by Proposition 2.6. It follows that $u^{*}=P_{\Omega}\left[u^{*}-\beta\left(u^{*}+\nabla f\left(u^{*}\right)\right)\right]$, that is to say $u^{*}$ is a solution of the variational inequality $\operatorname{VI}\left(\Omega, I+\nabla f J_{\lambda}\right)$. Let $\tilde{u}=P_{\Omega}\left[u-\beta\left(u+\nabla f J_{\lambda}(u)\right)\right]$, by using $u^{*}=J_{\lambda}\left(u^{*}\right)$ again, we can obtain the first inequality:

$$
\left\langle\tilde{u}-u^{*}, \beta\left(u^{*}+\nabla f J_{\lambda}\left(u^{*}\right)\right)\right\rangle \geqslant 0 .
$$

Since $u^{*} \in \Omega, \tilde{u}=P_{\Omega}\left[u-\beta\left(u+\nabla f J_{\lambda}(u)\right)\right]$, according to the property of the projection $P_{\Omega}$, we get the second inequality:

$$
\left\langle\tilde{u}-u^{*}, u-\beta\left(u+\nabla f J_{\lambda}(u)\right)-\tilde{u}\right\rangle \geqslant 0, \quad \forall u \in H .
$$

Under the assumption that $-\nabla f$ and $J_{\lambda}$ are nonexpansive, by Lemma $2.2, \beta\left(I+\nabla f J_{\lambda}\right)$ is $\frac{1}{2 \beta}$-ism, we have the third inequality:

$$
\left\langle\tilde{u}-u^{*}, \beta\left(I+\nabla f J_{\lambda}\right)(\tilde{u})-\beta\left(I+\nabla f J_{\lambda}\right)\left(u^{*}\right)\right\rangle \geqslant 0, \quad \forall u \in H .
$$

Inequalities (2.1), (2.2), (2.3) play an important role in projection and contraction methods. 


\section{Main results}

In this section, we first consider how to construct projection and contraction method for constrained convex minimization and the zero points of maximal monotone operator problem (1.5) and then we get the weak convergence theorem for the iterative algorithm.

For any $\beta>0$ and a current point $u_{k}, \tilde{u_{k}}$ is obtained by the projection

$$
\tilde{\mathfrak{u}_{k}}=P_{\Omega}\left[\mathfrak{u}_{k}-\beta\left(\mathfrak{u}_{k}+\nabla f J_{\lambda}\left(\mathfrak{u}_{k}\right)\right)\right]=P_{\Omega}\left[(1-\beta) \mathfrak{u}_{k}+\beta\left(-\nabla f J_{\lambda}\right)\left(\mathfrak{u}_{k}\right)\right] .
$$

By Proposition 2.6, the current point $u_{k}$ is a solution, if and only if $u_{k}=\tilde{u_{k}}$.

Definition 3.1. A non-negative function $\varphi(u, \tilde{u})$ is called to be an error metric function of the problem (1.5), if there exists $\delta>0$ such that

$$
\varphi(u, \tilde{u}) \geqslant \delta\|u-\tilde{u}\|,
$$

and $\varphi(u, \tilde{u})=0$, if and only if $\mathfrak{u}=\tilde{u}$.

Definition 3.2. A vector $d(u, \tilde{u})$ is called a profitable direction respect to the error metric function $\varphi(u, \tilde{u})$, if

$$
\left\langle u-u^{*}, d(u, \tilde{u})\right\rangle \geqslant \varphi(u, \tilde{u}),
$$

for all $u^{*}$ belong to the solution set $\Gamma$.

Remark 3.3. Assume $u^{*} \in \Gamma$ is a solution of problem (1.5), then $-d\left(u_{k}, \tilde{u_{k}}\right)$ is a drop direction of the function $\frac{1}{2}\left\|u-u^{*}\right\|^{2}$ at the point $u_{k}$. The following iterative scheme for problem (1.5), based on the projection and contraction methods which assure that the distance form the iteration points generated by the algorithm to a point of the solution set is strictly monotone decreasing.

Algorithm 3.4. $\mathfrak{u}_{k+1}=\mathfrak{u}_{k}-\alpha_{k} d\left(u_{k}, \tilde{u_{k}}\right)$, where $\alpha_{k}=\frac{\varphi\left(u_{k}, \tilde{u}_{k}\right)}{\left\|d\left(u_{k}, \tilde{u}_{k}\right)\right\|^{2}}$.

Thereinto, $d\left(u_{k}, \tilde{u_{k}}\right)$ and $\varphi\left(u_{k}, \tilde{u_{k}}\right)$ is constructed as follows: Adding (2.1), (2.2) and (2.3), we obtain

$$
\left\langle\tilde{u}-u^{*}, u-\tilde{u}-\beta\left[\left(I+\nabla f J_{\lambda}\right)(u)-\left(I+\nabla f J_{\lambda}\right)(\tilde{u})\right]\right\rangle \geqslant 0, \quad \forall u \in H .
$$

Let

$$
d(u, \tilde{u})=u-\tilde{u}-\beta\left[\left(I+\nabla f J_{\lambda}\right)(u)-\left(I+\nabla f J_{\lambda}\right)(\tilde{u})\right],
$$

and

$$
\varphi(u, \tilde{u})=\langle u-\tilde{u}, d(u, \tilde{u})\rangle
$$

It follows that for all $u \in H$,

$$
\left\langle u-u^{*}, d(u, \tilde{u})\right\rangle \geqslant \varphi(u, \tilde{u}) .
$$

Since $\beta\left(I+\nabla f J_{\lambda}\right)$ is $\frac{1}{2 \beta}$-ism, one can get

$$
\frac{1}{2 \beta}\left\|\beta\left(I+\nabla f J_{\lambda}\right)\left(u_{1}\right)-\beta\left(I+\nabla f J_{\lambda}\right)\left(u_{2}\right)\right\|^{2} \leqslant\left\langle\beta\left(I+\nabla f J_{\lambda}\right)\left(u_{1}\right)-\beta\left(I+\nabla f J_{\lambda}\right)\left(u_{2}\right), u_{1}-u_{2}\right\rangle,
$$

hence

$$
\left\|\beta\left(I+\nabla f J_{\lambda}\right)\left(u_{1}\right)-\beta\left(I+\nabla f J_{\lambda}\right)\left(u_{2}\right)\right\| \leqslant 2 \beta\left\|u_{1}-u_{2}\right\| .
$$

It follows that

$$
\begin{aligned}
\varphi(u, \tilde{u}) & =\langle u-\tilde{u}, d(u, \tilde{u})\rangle \\
& =\|u-\tilde{u}\|^{2}-\left\langle u-\tilde{u}, \beta\left(I+\nabla f J_{\lambda}\right)(u)-\beta\left(I+\nabla f J_{\lambda}\right)(\tilde{u})\right\rangle \\
& \geqslant(1-2 \beta)\|u-\tilde{u}\|^{2} .
\end{aligned}
$$


That is to say, $\varphi(u, \tilde{u})$ is an error metric function of the problem $(1.5)$ and $d(u, \tilde{u})$ is a profitable direction respect to the error metric function $\varphi(u, \tilde{u})$.

Next, we prove that the sequence generated by Algorithm 3.4 converges weakly to a point of the solution set $\Gamma$ of problem (1.5).

Theorem 3.5. Let $\mathrm{H}$ be a real Hilbert space and let $\Omega$ be a nonempty closed convex subset of $\mathrm{H}$. Let $\mathrm{A}$ be a maximal monotone operator on $\mathrm{H}$ such that the domain of $\mathrm{A}$ is included in $\Omega$. Let $\mathrm{J}_{\lambda}=(\mathrm{I}+\lambda \mathrm{A})^{-1}$ be the resolvent of $\mathrm{A}$ for $\lambda>0$. Let $\mathrm{f}$ be a real-valued convex function of $\Omega$ into $\mathrm{R}$ and $\mathrm{I}+\nabla \mathrm{f}$ is $\mathrm{v}$-inverse strongly monotone. Assume that the solution set $\Gamma$ of the problem (1.5) is consistent. Let the sequence $\left\{\mathfrak{u}_{\mathrm{k}}\right\}$ be generated by Algorithm 3.4, i.e.,

$$
u_{k+1}=u_{k}-\alpha_{k} d\left(u_{k}, \tilde{u_{k}}\right),
$$

where

$$
\begin{aligned}
\tilde{u_{k}} & =P_{\Omega}\left[u_{k}-\beta\left(I+\nabla f J_{\lambda}\right)\left(u_{k}\right)\right], \\
d\left(u_{k}, \tilde{u_{k}}\right) & =u_{k}-\tilde{u_{k}}-\beta\left[\left(I+\nabla f J_{\lambda}\right)\left(u_{k}\right)-\left(I+\nabla f J_{\lambda}\right)\left(\tilde{u_{k}}\right)\right],
\end{aligned}
$$

and

$$
\varphi\left(u_{k}, \tilde{u_{k}}\right)=\left\langle u_{k}-\tilde{u_{k}}, d\left(u_{k}, \tilde{u_{k}}\right)\right\rangle, \quad \alpha_{k}=\frac{\varphi\left(u_{k}, \tilde{u_{k}}\right)}{\left\|d\left(u_{k}, \tilde{u_{k}}\right)\right\|^{2}}, \quad \beta \in\left(0, \frac{1}{2}\right) .
$$

Then the sequence $\left\{u_{k}\right\}$ converges weakly to a point of the solution set $\Gamma$ of problem (1.5).

Proof. Assume that $\mathrm{u}^{*} \in \Gamma$, one can get

$$
\begin{aligned}
\left\|u_{k+1}-u^{*}\right\|^{2} & =\left\|u_{k}-u^{*}-\alpha_{k} d\left(u_{k}, \tilde{u_{k}}\right)\right\|^{2} \\
& =\left\langle u_{k}-u^{*}-\alpha_{k} d\left(u_{k}, \tilde{u_{k}}\right), u_{k}-u^{*}-\alpha_{k} d\left(u_{k}, \tilde{u_{k}}\right)\right\rangle \\
& =\left\|u_{k}-u^{*}\right\|^{2}-2\left\langle u_{k}-u^{*}, \alpha_{k} d\left(u_{k}, \tilde{u_{k}}\right)\right\rangle+\alpha_{k}^{2}\left\|d\left(u_{k}, \tilde{u_{k}}\right)\right\|^{2} \\
& \leqslant\left\|u_{k}-u^{*}\right\|^{2}-\alpha_{k} \varphi\left(u_{k}, \tilde{u_{k}}\right) \\
& =\left\|u_{k}-u^{*}\right\|^{2}-\alpha_{k}^{2}\left\|d\left(u_{k}, \tilde{u_{k}}\right)\right\|^{2} \\
& =\left\|u_{k}-u^{*}\right\|^{2}-\left\|u_{k+1}-u_{k}\right\|^{2} .
\end{aligned}
$$

Hence, $\left\|\mathfrak{u}_{k+1}-\mathfrak{u}^{*}\right\|$ is a decreasing sequence, $\lim _{k \rightarrow \infty}\left\|\mathfrak{u}_{k}-u^{*}\right\|$ exists and

$$
\Sigma_{\mathrm{k}=0}^{\infty}\left\|\mathfrak{u}_{\mathrm{k}+1}-\mathfrak{u}_{\mathrm{k}}\right\|^{2} \leqslant\left\|\mathfrak{u}_{0}-\mathfrak{u}^{*}\right\|^{2} .
$$

On the other hand,

$$
\begin{aligned}
\left\|u_{k+1}-u^{*}\right\|^{2} & \leqslant\left\|u_{k}-u^{*}\right\|^{2}-\alpha_{k} \varphi\left(u_{k}, \tilde{u_{k}}\right) \\
& \leqslant\left\|u_{k}-u^{*}\right\|^{2}-\alpha_{k}(1-2 \beta)\left\|u_{k}-\tilde{u_{k}}\right\|^{2},
\end{aligned}
$$

and

$$
\begin{aligned}
2 \varphi\left(u_{k}, \tilde{u_{k}}\right)-\left\|d\left(\tilde{u_{k}}, \tilde{u_{k}}\right)\right\|^{2} & =\left\langle d\left(u_{k}, \tilde{u_{k}}\right), 2\left(u_{k}-\tilde{u_{k}}\right)-d\left(u_{k}, \tilde{u_{k}}\right)\right\rangle \\
& =\left\|u_{k}-\tilde{u_{k}}\right\|^{2}-\left\|\beta\left(I+\nabla f J_{\lambda}\right)\left(u_{k}\right)-\beta\left(I+\nabla f J_{\lambda}\right)\left(\tilde{u_{k}}\right)\right\|^{2} \\
& \geqslant\left\|u_{k}-\tilde{u_{k}}\right\|^{2}-4 \beta^{2}\left\|u_{k}-\tilde{u_{k}}\right\|^{2}>0 .
\end{aligned}
$$

That is to say $\alpha_{k}>\frac{1}{2}$.

One can get

$$
\lim _{k \rightarrow \infty}\left\|u_{k}-\tilde{u_{k}}\right\|=0
$$

Hence if $\mathfrak{u}_{k_{n}} \rightarrow \hat{u}$, then $\lim _{n \rightarrow \infty}\left\|\mathfrak{u}_{k_{n}}-\mathfrak{u}_{k_{n}}\right\|=0$, by Lemma 2.3, $\hat{u} \in \Gamma$. In addition, for any $\mathfrak{u}^{*} \in \Gamma$, $\lim _{k \rightarrow \infty}\left\|u_{k}-u^{*}\right\|$ exists, by using Lemma 2.4 , we can get $u_{k}$ converges weakly to a point of the solution set $\Gamma$ of problem (1.5). 


\section{Applications}

In this section, we will give some applications which are useful in nonlinear analysis and optimization.

Firstly, recall the equilibrium problem, assume $\Omega$ is a nonempty closed convex subset of real Hilbert spaces $H, F: \Omega \times \Omega \longrightarrow R$ is a bifunction of $\Omega \times \Omega$ into $R$ the set of real numbers. The equilibrium problem (EP) is to find $z \in \Omega$ such that

$$
\mathrm{F}(z, \mathrm{y}) \geqslant 0, \quad \forall \mathrm{y} \in \Omega .
$$

Lots of problems in physics, optimizations and economics can be reduced to find a solution of the EP. For more details we refer the reader to [6, 11, 18, 25]. For solving the EP, let us assume that $F$ satisfies the following conditions:

(i) for all $x \in \Omega, F(x, x)=0$;

(ii) for all $x, y \in \Omega, F(x, y)+F(y, x) \leqslant 0$;

(iii) for any $x, y, z \in \Omega, \lim _{t \rightarrow 0} F(t z+(1-t) x, y) \leqslant F(x, y)$;

(iv) for any $x \in \Omega, y \mapsto F(x, y)$ is convex and lower semi-continuous.

We use $\Gamma_{2}$ to denote the solution set of EP and assume consistency of EP so that $\Gamma_{2}$ is nonempty. The following proposition was given by Combettes and Hirstoaga [6].

Proposition 4.1. Assume a bifunction $\mathrm{F}: \Omega \times \Omega \longrightarrow \mathrm{R}$ satisfies (i)-(iv). For $\lambda>0, x \in \mathrm{H}$, define mapping $\mathrm{J}_{\lambda}: \mathrm{H} \longrightarrow \Omega$ as below:

$$
\mathrm{J}_{\lambda}(\mathrm{x})=\left\{z \in \Omega, \mathrm{F}(z, \mathrm{y})+\frac{1}{\lambda}\langle\mathrm{y}-z, z-x\rangle \geqslant 0, \forall \mathrm{y} \in \Omega\right\} .
$$

Then, we have:

$\mathrm{J}_{\lambda}$ is single-valued, $\mathrm{J}_{\lambda}$ is fne; $\mathrm{Fix}\left(\mathrm{J}_{\lambda}\right)=\Gamma_{2}$ and $\Gamma_{2}$ the solution set of EP is closed and convex.

Using Theorem 3.5, we have the following result:

Theorem 4.2. Let $\mathrm{H}$ be a real Hilbert space and let $\Omega$ be a nonempty closed convex subset of $\mathrm{H} . \mathrm{F}: \Omega \times \Omega \longrightarrow R$ is a bifunction of $\Omega \times \Omega$ into $\mathrm{R}$ satisfies (i)-(iv). Let $\mathrm{J}_{\lambda}$ be defined as in Proposition 4.1, $\mathrm{f}$ be a real-valued convex function of $\Omega$ into $R$, and the gradient be av. Assume $\Gamma_{1} \cap \Gamma_{2} \neq \emptyset$. For an arbitrary point $u_{0} \in H$ the sequence $\left\{u_{k}\right\}$ is generated by the iterative algorithm

$$
u_{k+1}=u_{k}-\alpha_{k} d\left(u_{k}, \tilde{u_{k}}\right) \text {, }
$$

where

$$
\begin{aligned}
\tilde{u_{k}} & =P_{\Omega}\left[u_{k}-\beta\left(I+\nabla f J_{\lambda}\right)\left(u_{k}\right)\right], \\
d\left(u_{k}, \tilde{u_{k}}\right) & =u_{k}-\tilde{u_{k}}-\beta\left[\left(I+\nabla f J_{\lambda}\right)\left(u_{k}\right)-\left(I+\nabla f J_{\lambda}\right)\left(\tilde{u_{k}}\right)\right],
\end{aligned}
$$

and

$$
\varphi\left(u_{k}, \tilde{u_{k}}\right)=\left\langle u_{k}-\tilde{u_{k}}, d\left(u_{k}, \tilde{u_{k}}\right)\right\rangle, \quad \alpha_{k}=\frac{\varphi\left(\tilde{u_{k}}, \tilde{u_{k}}\right)}{\left\|d\left(u_{k}, \tilde{u_{k}}\right)\right\|^{2}}, \quad \beta \in\left(0, \frac{1}{2}\right) .
$$

Then the sequence $\left\{u_{k}\right\}$ converges weakly to a point of the set $\Gamma_{1} \bigcap \Gamma_{2}$ which is a solution of the regularized minimization problem (1.2) and the EP (4.1).

Secondly, recall the split feasibility and split equality problem. In 1994, Censor and Elfving [5] introduced the split feasibility problem (SFP). Let $\mathrm{C}$ and $\mathrm{Q}$ be nonempty closed convex subset of real Hilbert spaces $\mathrm{H}_{1}$ and $\mathrm{H}_{2}$, respectively, and let $A: \mathrm{H}_{1} \rightarrow \mathrm{H}_{2}$ be a bounded linear operator. Recall that the split feasibility problem (SFP) is to find a point $u$ satisfying the property:

$$
u \in C, \quad A u \in Q,
$$


if such point exists. Ever since its introduction, SFP attracted many authors' attention due to its applications in signal processing [5]. Various algorithms have been invented to solve it (see [1, 2, 19, 27, 29-31]).

Recently, Moudafi [16] proposed a new split equality problem (SEP): Let $\mathrm{H}_{1}, \mathrm{H}_{2}, \mathrm{H}_{3}$ be real Hilbert spaces, $\mathrm{C} \subseteq \mathrm{H}_{1}, \mathrm{Q} \subseteq \mathrm{H}_{2}$ be two nonempty closed convex sets and let $A: \mathrm{H}_{1} \rightarrow \mathrm{H}_{3}, \mathrm{~B}: \mathrm{H}_{2} \rightarrow \mathrm{H}_{3}$ be two bounded linear operators. Find $x \in C, y \in Q$ satisfying

$$
A x=B y .
$$

When $B=I$, SEP reduces to the well-known SFP. We use $\Gamma_{3}$ to denote the solution set of SEP and assume consistency of SEP so that $\Gamma_{3}$ is nonempty closed and convex.

In the paper [16], Moudafi gave the alternating CQ-algorithm and relaxed alternating CQ-algorithm iterative algorithm for solving the split equality problem. In the paper [22], we used the well-known Tychonov regularization and got some algorithms converging strongly to the minimum-norm solution of the SEP. form

Let $\Omega=\mathrm{C} \times \mathrm{Q}$ in $\mathrm{H}=\mathrm{H}_{1} \times \mathrm{H}_{2}$, define $\mathrm{G}: \mathrm{H} \rightarrow \mathrm{H}_{3}$ by $\mathrm{G}=[\mathrm{A},-\mathrm{B}]$, then $\mathrm{G}^{*} \mathrm{G}: \mathrm{H} \rightarrow \mathrm{H}$ has the matrix

$$
G^{*} G=\left[\begin{array}{cc}
A^{*} A & -A^{*} B \\
-B^{*} A & B^{*} B
\end{array}\right]
$$

The original problem can now be reformulated as finding $w=(x, y) \in \Omega$ with $G w=0$, or more generally, minimizing the function $\|\mathrm{G} w\|$ over $w \in \Omega$. Therefore solving SEP is equivalent to solving the following minimization problem

$$
\min _{w \in \Omega} f(w)=\frac{1}{2}\|\mathrm{G} w\|^{2},
$$

which is in general ill-posed. A classical way to deal with such a possibly ill-posed problem is the wellknown Tychonov regularization:

$$
\min _{w \in \Omega} f_{\alpha}(w)=\frac{\alpha}{2}\|G w\|^{2}+\frac{1}{2}\|w\|^{2} .
$$

We can get that $f_{\alpha}$ is strictly convex, coercive and differentiable with gradient

$$
\nabla f_{\alpha}(w)=\left(I+\alpha G^{*} G\right) w .
$$

Remark 4.3. If $0<\alpha<\frac{1}{\|\mathrm{G}\|^{2}}$, then $\alpha \mathrm{G}^{*} \mathrm{G}$ is an av operator. In fact, for all $x, y \in \mathrm{H}$,

$$
\begin{aligned}
\left\|\left(I-\alpha G^{*} G\right) x-\left(I-\alpha G^{*} G\right) y\right\|^{2} & =\|x-y\|^{2}+\alpha^{2}\left\|G^{*} G(x-y)\right\|^{2}-2 \alpha\|G(x-y)\|^{2} \\
& \leqslant\|x-y\|^{2}-\alpha\|G(x-y)\|^{2} \\
& =\left\langle\left(I-\alpha G^{*} G\right)(x-y), x-y\right\rangle .
\end{aligned}
$$

That is to say $I-\alpha G^{*} G$ is fne. So, $\alpha G^{*} G$ is av operator.

The structure of the error metric function and the profitable direction are as follows:

$$
\begin{gathered}
\tilde{u_{k}}=P_{\Omega}\left[u_{k}-\beta\left(I+\alpha G^{*} G\right)\left(u_{k}\right)\right]=P_{\Omega}\left[(1-\beta) u_{k}-\alpha \beta G^{*} G u_{k}\right], \\
\begin{aligned}
d\left(u_{k}, \tilde{u_{k}}\right) & =u_{k}-\tilde{u_{k}}-\beta\left[\left(I+\alpha G^{*} G\right)\left(u_{k}\right)-\left(I+\alpha G^{*} G\right)\left(\tilde{u_{k}}\right)\right] \\
& =(1-\beta)\left(u_{k}-\tilde{u_{k}}\right)-\alpha \beta G^{*} G\left(u_{k}-\tilde{u_{k}}\right),
\end{aligned}
\end{gathered}
$$

and

$$
\begin{gathered}
\varphi\left(\tilde{u}_{k}, \tilde{u_{k}}\right)=\left\langle u_{k}-\tilde{u_{k}}, d\left(\tilde{u_{k}}, \tilde{u_{k}}\right)\right\rangle \\
=(1-\beta)\left\|u_{k}-\tilde{u_{k}}\right\|^{2}-\alpha \beta\left\|G\left(u_{k}-\tilde{u_{k}}\right)\right\|^{2}, \\
\alpha_{k}=\frac{\varphi\left(u_{k}, \tilde{u_{k}}\right)}{\left\|d\left(u_{k}, \tilde{u_{k}}\right)\right\|^{2}}=\frac{(1-\beta)\left\|u_{k}-\tilde{u_{k}}\right\|^{2}-\alpha \beta\left\|G\left(u_{k}-\tilde{u_{k}}\right)\right\|^{2}}{(1-\beta)^{2}\left\|\tilde{u_{k}}-\tilde{u_{k}}\right\|^{2}-\alpha^{2} \beta^{2}\left\|G * G\left(u_{k}-\tilde{u_{k}}\right)\right\|^{2}-2 \alpha \beta(1-\beta)\left\|G\left(\tilde{u_{k}}-\tilde{u_{k}}\right)\right\|^{2}} .
\end{gathered}
$$

According to Theorem 3.5, we can get the following result. 
Theorem 4.4. For an arbitrary point $\mathrm{u}_{0}=\left(\mathrm{x}_{0}, \mathrm{y}_{0}\right) \in \mathrm{H}=\mathrm{H}_{1} \times \mathrm{H}_{2}$, the sequence $\left\{\mathrm{u}_{\mathrm{k}}\right\}=\left\{\left(\mathrm{x}_{\mathrm{k}}, \mathrm{y}_{\mathrm{k}}\right)\right\}$ is generated by the iterative algorithm

$$
\mathfrak{u}_{k+1}=\mathfrak{u}_{k}-\alpha_{k}\left[(1-\beta)\left(\mathfrak{u}_{k}-\tilde{u_{k}}\right)-\alpha \beta G^{*} G\left(u_{k}-\tilde{u_{k}}\right)\right],
$$

where

$$
\begin{aligned}
& \tilde{u_{k}}=P_{\Omega}\left[(1-\beta) u_{k}-\alpha \beta G^{*} G u_{k}\right], \beta \in\left(0, \frac{1}{2}\right), \quad 0<\alpha<\frac{1}{\|G\|^{2}}, \\
& \alpha_{k}=\frac{(1-\beta)\left\|u_{k}-\tilde{u_{k}}\right\|^{2}-\alpha \beta\left\|G\left(u_{k}-\tilde{u_{k}}\right)\right\|^{2}}{(1-\beta)^{2}\left\|u_{k}-\tilde{u_{k}}\right\|^{2}-\alpha^{2} \beta^{2}\left\|G * G\left(u_{k}-\tilde{u_{k}}\right)\right\|^{2}-2 \alpha \beta(1-\beta)\left\|G\left(u_{k}-\tilde{u_{k}}\right)\right\|^{2}} .
\end{aligned}
$$

Then the sequence $\left\{\mathfrak{u}_{\mathrm{k}}\right\}$ converges weakly to the minimum-norm solution of the SEP (4.2).

Remark 4.5. In Theorem 4.4, let $G=[A,-I]$, then the sequence $\left\{\mathfrak{u}_{k}\right\}$ converges weakly to the minimumnorm solution of the SFP.

Finally, we consider the SEP and the EP at the same time. Assume that intersection of solution of EP and SEP is not a empty set. By Theorem 3.5, we have:

Theorem 4.6. Let $\mathrm{H}_{1}, \mathrm{H}_{2}, \mathrm{H}_{3}$ be real Hilbert spaces, $\mathrm{C} \subseteq \mathrm{H}_{1}, \mathrm{Q} \subseteq \mathrm{H}_{2}$ be two nonempty closed convex sets and let A : $\mathrm{H}_{1} \rightarrow \mathrm{H}_{3}, \mathrm{~B}: \mathrm{H}_{2} \rightarrow \mathrm{H}_{3}$ be two bounded linear operators. Let $\mathrm{H}=\mathrm{H}_{1} \times \mathrm{H}_{2}, \Omega=\mathrm{C} \times \mathrm{Q}, \mathrm{F}: \Omega \times \Omega \longrightarrow \mathrm{R}$ be a bifunction of $\Omega \times \Omega$ into $\mathrm{R}$ satisfying (i)-(iv). Let $\mathrm{J}_{\lambda}$ be defined as in Proposition 4.1. Assume $\Gamma_{2} \cap \Gamma_{3}$, the intersection of solution of EP and SEP is not an empty set. For an arbitrary point $\mathfrak{u}_{0} \in \mathrm{H}$, the sequence $\left\{\mathfrak{u}_{\mathrm{k}}\right\}$ is generated by the iterative algorithm

$$
u_{k+1}=u_{k}-\alpha_{k} d\left(u_{k}, \tilde{u_{k}}\right) \text {, }
$$

where

$$
\tilde{u_{k}}=P_{\Omega}\left[u_{k}-\beta\left(I+\alpha G^{*} G J_{\lambda}\right)\left(u_{k}\right)\right], \quad d\left(u_{k}, \tilde{u_{k}}\right)=u_{k}-\tilde{u_{k}}-\beta\left[\left(I+\alpha G^{*} G J_{\lambda}\right)\left(u_{k}\right)-\left(I+\alpha G^{*} G J_{\lambda}\right)\left(\tilde{u_{k}}\right)\right],
$$

and

$$
\varphi\left(\mathfrak{u}_{k}, \tilde{u_{k}}\right)=\left\langle u_{k}-\tilde{u_{k}}, d\left(u_{k}, \tilde{u_{k}}\right)\right\rangle, \quad \alpha_{k}=\frac{\varphi\left(u_{k}, \tilde{u_{k}}\right)}{\left\|d\left(\tilde{u_{k}}, \tilde{u_{k}}\right)\right\|^{2}}, \quad \beta \in\left(0, \frac{1}{2}\right), \quad 0<\alpha<\frac{1}{\|G\|^{2}} .
$$

Then the sequence $\left\{u_{k}\right\}$ converges weakly to a point of the set $\Gamma_{2} \bigcap \Gamma_{3}$.

\section{Acknowledgment}

This research is supported by NSFC Grants No:11301379; No:11226125; No:11671167.

\section{References}

[1] C. Byrne, Iterative oblique projection onto convex sets and the split feasibility problem, Inverse Problems, 18 (2002), 441-453. 4

[2] C. Byrne, A unified treatment of some iterative algorithms in signal processing and image reconstruction, Inverse Problems, 20 (2004), 103-120. 2.2, 4

[3] X.-J. Cai, G.-Y. Gu, B.-S. He, On the $\mathrm{O}(1 / \mathrm{t})$ convergence rate of the projection and contraction methods for variational inequalities with Lipschitz continuous monotone operators, Comput. Optim. Appl., 57 (2014), 339-363. 1

[4] L.-C. Ceng, Q. H. Ansari, J.-C. Yao, Extragradient-projection method for solving constrained convex minimization problems, Numer. Algebra Control Optim., 1 (2011), 341-359. 1

[5] Y. Censor, T. Elfving, A multiprojection algorithm using Bregman projections in a product space, Numer. Algorithms, 8 (1994), 221-239. 4

[6] P. L. Combettes, S. A. Hirstoaga, Equilibrium programming in Hilbert spaces, J. Nonlinear Convex Anal., 6 (2005), 117-136. 4

[7] Q. L. Dong, J. Yang, H. B. Yuan, The projection and contraction algorithm for solving variational inequality problems in Hilbert spaces, to appear in J. Nonlinear Convex Anal. 1 
[8] J. Eckstein, D. P. Bertsekas, On the Douglas-Rachford splitting method and the proximal point algorithm for maximal monotone operators, Math. Programming, 55 (1992), 293-318. 1

[9] J. Eckstein, B. F. Svaiter, A family of projective splitting methods for the sum of two maximal monotone operators, Math. Program., 111 (2008), 1173-1199. 1

[10] H. W. Engl, M. Hanke, A. Neubauer, Regularization of inverse problems, Mathematics and its Applications, Kluwer Academic Publishers Group, Dordrecht, (1996). 2.5

[11] S. D. Flåm, A. S. Antipin, Equilibrium programming using proximal-like algorithms, Math. Programming, 78 (1997), 29-41. 4

[12] K. Goebel, W. A. Kirk, Topics in metric fixed point theory, Cambridge Studies in Advanced Mathematics, Cambridge University Press, Cambridge, (1990). 2.3

[13] K. Geobel, S. Reich, Uniform convexity, hyperbolic geometry, and nonexpansive mappings, Monographs and Textbooks in Pure and Applied Mathematics, Marcel Dekker, Inc., New York, (1984). 2.3

[14] B.-S. He, A class of projection and contraction methods for monotone variational inequalities, Appl. Math. Optim., 35 (1997), 69-76. 1

[15] P.-L. Lions, Une méthode itérative de résolution d'une inéquation variationnelle, (French) Israel J. Math., 31 (1978), 204-208. 1

[16] A. Moudafi, A relaxed alternating CQ-algorithm for convex feasibility problems, Nonlinear Anal., 79 (2013), 117-121. 4

[17] G. B. Passty, Ergodic convergence to a zero of the sum of monotone operators in Hilbert space, J. Math. Anal. Appl., 72 (1979), 383-390. 1

[18] X. L. Qin, Y. J. Cho, S. M. Kang, Convergence analysis on hybrid projection algorithms for equilibrium problems and variational inequality problems, Math. Model. Anal., 14 (2009), 335-351. 4

[19] B. Qu, N.-H. Xiu, A note on the CQ algorithm for the split feasibility problem, Inverse Problems, 21 (2005), 1655-1665. 4

[20] F. Schöpfer, T. Schuster, A. K. Louis, An iterative regularization method for the solution of the split feasibility problem in Banach spaces, Inverse Problems, 24 (2008), 20 pages. 2.4

[21] L. Y. Shi, R.-D. Chen, Y.-J. Wu, Iterative algorithms for finding the zeroes of sums of operators, J. Inequal. Appl., 2014 (2014), 16 pages. 1

[22] L. Y. Shi, R.-D. Chen, Y.-J. Wu, Strong convergence of iterative algorithms for the split equality problem, J. Inequal. Appl., 2014 (2014), 19 pages. 4

[23] D. Sun, A class of iterative methods for solving nonlinear projection equations, J. Optim. Theory Appl., 91 (1996), 123-140. 1

[24] W. Takahashi, Totsu kaiseki to fudōten kinji, (Japanese) [[Convex analysis \& approximation of fixed points]] Sūrikaiseki Shiriizu [Mathematical Analysis Series], Yokohama Publishers, Yokohama, (2000). 1, 2

[25] S. Takahashi, W. Takahashi, Viscosity approximation methods for equilibrium problems and fixed point problems in Hilbert spaces, J. Math. Anal. Appl., 331 (2007), 506-515. 4

[26] P. Tseng, A modified forward-backward splitting method for maximal monotone mappings, SIAM J. Control Optim., 38 (2000), 431-446. 1

[27] H.-K. Xu, A variable Krasnoselski-Mann algorithm and the multiple-set split feasibility problem, Inverse Problems, 22 (2006), 2021-2034. 4

[28] H.-K. Xu, Iterative methods for the split feasibility problem in infinite-dimensional Hilbert spaces, Inverse Problems, 26 (2010), 105-128. 1

[29] H.-K. Xu, Averaged mappings and the gradient-projection algorithm, J. Optim. Theory Appl., 150 (2011), 360-378. 1, 4

[30] Q.-Z. Yang, The relaxed CQ algorithm solving the split feasibility problem, Inverse Problems, 20 (2004), 1261-1266.

[31] Q.-Z. Yang, J.-L. Zhao, Generalized KM theorems and their applications, Inverse Problems, 22 (2006), 833-844. 4 